\title{
3D pore analysis of sedimentary rocks
}

\author{
E. Vergés ${ }^{a}$ D. Tost ${ }^{a}$. D. Ayalaa ${ }^{a}$ E. Ramos ${ }^{b}$ and S.Grau \\ a CREB, UPC (Polytechnical University Catalonia)/ IBEC (Institute for Bioengineering Catalonia) \\ Barcelona, Spain \\ ${ }^{b}$ Grup de Geodinàmica i Anàlisis de Conques - GEOMODELS. Dep. d'Estratigrafia, Paleontologìa \\ i Geociències Marines, Universitat de Barcelona, c/ Martí i Franqués, s/n. E-08028, Barcelona, \\ Spain
}

(everges, dani, dolorsa, sgrau)@lsi.upc.edu

emilio.ramos@ub.edu

\begin{abstract}
A 3D representation of the internal structure and fabric of sedimentary rocks is of paramount interest to evaluate their structural parameters such as porosity, pore-size distribution and permeability. The classical experimental technique to evaluate the pore space volume and pore size distribution is the Mercury Intrusion Porosimetry (MIP). Computer-based methods use 3D imaging technologies such as Computer Tomography (CT) scanned images to construct and evaluate a 3D virtual representation of the internal pore distribution. In this work, based on a three samples set of sandstone, we apply two numerical (computer-based) methods in order to reconstruct and analyse the internal pore network, and compare it with the results obtained by MIP analysis. The first numerical method performs a virtual simulation of MIP. The second one obtains a graph of pores using a sphere-filling based approach. For all methods, we compute the global porosity and the pore-size distribution. Moreover, with the numerical methods, we obtain the total porosity and a graph representing the pore space that can be visualized with 3D illustration techniques.
\end{abstract}

Key words:

Porosimetry, 3D pore reconstruction, Virtual measurements. Illustrative visualization. 


\section{Introduction}

The geometrical and topological representation of the internal structure of rocks is necessary for the evaluation of their physical properties. It provides means of simulating transport and distribution of fluids such as water, oil or gas through their pore framework. The classical experimental methods to measure porosity use Mercury Intrusion Porosimetry (MIP) based on the capillarity law governing penetration of non-wetting liquid into porous regions. With this technique, mercury is intruded at different pressures into the pore space. For each pressure $P$, the throat diameter (D) can be calculated according to Washburn Equation: $D=-(4 \mathrm{WC} Y \cos \varphi) / P$, where $\gamma$ is the surface tension, $\varphi$ is the contact angle and WC is the Washburn constant. The pore space is thus approximated as a set of cylinders with a diameter corresponding to the throat of intrusion and a volume equivalent to that of the intruded mercury.

New non-destructive computer-based methods are now being used to evaluate structural parameters. They use images captured with different imaging technology such as 3D microscopy, Computer Tomography (CT or $\mu \mathrm{CT}$ ), and synchrotron light to construct 3D virtual models of the samples and apply numerical evaluation processes. An advantage of these methods is that they can provide not only the size distribution of the pores inside the material but also a geometrical and topological representation of the network of pores. Numerical porosimetry methods have been applied to soil, sediment and sedimentary rock samples.

With CT images, porosity can be computed directly from the CT values (Taud et al., 2005) or by simple voxel counting (Tuan and Hutmacher, 2005). The pore space topology can be represented in several ways. A cylinder network model is used by Deleure et al. (2003) to simulate MIP on soil samples. Sillin et al. (2004) obtained a stick-and-ball graph by, first, computing a 2D skeleton, and, next, filling it with spheres. A 1D skeleton is used by Liang et al. (2000), Lindquist et al. (2000) and Oren and Bakke (2003). This skeleton is taken as an initial graph that partitions the pore space into pores centred at the graph vertices. The approach presented by Monga et al. (2007) obtains graphs of cylinders and bowls but, instead of filling the pore space, it approximates it with a controlled error. Moreover, there are approaches based on granulometry that perform successive morphological openings such as the methods proposed by Vogel et al. (2005) and Cnudde et al. (2009). In this second work, a comparison of granulometry-based results with data obtained from MIP and water absorption under vacuum is presented. Finally, other works are based on artificial, synthetic samples: Stroeven et al. (2006) design a virtual concrete with random particle addition, and, Acharya et al. (2004) build a virtual hydraulic pore network with pores of random sizes equally spaced. Recently, two numerical methods have been developed by our team: the first one, Virtual Porosimeter, simulates virtually how the MIP works (Vergés et al., 2008 a), and the other one, Pore Topology Tracker, applies a sphere-based approach to detect different-size cavities into the porous region (Vergés et al, 2008 b).

The goal of this paper is to compare the porosity measurements of sandstone samples that can be obtained with numerical methods and those provided by MIP. For this, we have obtained the porosity and pore-size 
distribution of three sandstone samples with MIP, we have got a set of $\mu$ CT scanned images of each sample and constructed a virtual 3D model of each. Next, we have applied the two numerical methods developed by our group to these 3D models in order to compute the total and effective porosity and the pore-size distribution. The analysis of the numerical results is complemented with a perceptual evaluation of the network that we obtain by creating several views of it and navigating though it. Finally, we compare and discuss the results obtained with MIP and the numerical methods.

\section{Materials and Methodology}

This study is based on three sandstone samples labelled as DS10, DS11 and DS12. The samples were collected from the Hawaz Formation, an oil bearing unit, Middle Ordovician in age, which widely crops out along the borders of the Murzuq Basin, in southern Libya (Ramos et al, 2006).

The work-flow includes the following steps:

1) Samples collection and preparation for thin sections and MIP and $\mu \mathrm{CT}$ tests

2) Petrographic study and analysis

3) MIP analysis

4) $\mu$ CT scanning

5) Construction of a voxel model from the 3D $\mu$ CT scanned images

6) Two-level segmentation of the model into solid region and porous region

7) Application of the Virtual Porosimeter numerical method

8) Application of the Pore Topology Tracker numerical method

9) Generation of illustrative visualizations

10) Results comparison

\subsection{Petrography}

Thin sections were studied by using a Carl-Zeiss AXIOPHOT petrographic microscope. Following the Gazzi-Dickinson method (Ingersoll et al., 1984) 150 points were counted for each thin section. The studied samples are fine to very fine-grained sandstones, with grain size ranging between 120 and $150 \mu \mathrm{m}$, and have a moderate to good sorting. Quartz grains are the major component for the three samples, which contain scarce amounts of other detrital components, including matrix (Figure 1); diagenetical processes and components are limited to cementation and compaction (Abouesa and Morad, 2009). As a consequence, the porosity of the studied samples is mainly formed by a network of open, intergranular pores displaying a relatively homogeneous poresize distribution, which in turn were primarily controlled by the size and sorting of the detrital grains, and secondarily reduced by cement precipitation and mechanical compaction. Diagenetical dissolution processes are infrequent.

\subsection{Mercury Intrusion Porosimetry}

Samples for MIP analysis were cut in cubes of approximately $15 \times 15 \times 15 \mathrm{~mm}$ each. We reserved some cubes for the Micro-CT scanning. The MIP analysis followed two steps: a low pressure and a high pressure mercury intrusion. The low pressure test was done using a QUANTQCHROME Autoscan Filling Aparatus 
working under a pressure range of 17 to $162 \mathrm{KPa}$, suitable for measuring pore sizes larger than $2 \cdot \mu \mathrm{m}$ in diameter. The high pressure test was done using the QUANTQCHROME Autoscan-33 working under a pressure range of 0.1 to 226 $\mathrm{MPa}$, suitable for measuring pore sizes from 0.01 to $2 \mu \mathrm{m}$ in diameter.

\subsection{Micro-CT scanned datasets}

We used the reserved sample cubes that were not processed by MIP. Otherwise, the mercury that had flood into the samples would have produced imaging artefacts. We could not perform the scanning first and then the MIP analysis on the same piece of sample, because the $\mu \mathrm{CT}$ device can process very small pieces of material only, too tiny to be processed by MIP. However, the material was very homogeneous, and the MIP analyses of different cubes gave the same results. Therefore, we believe that the $\mu \mathrm{CT}$ analyses are comparable with those of MIP, although they do not correspond to the exact same piece of material.

The $\mu \mathrm{CT}$ imaging was done in Trabeculae (www.trabbeculae.com) using a SkyScan 1172 and a reconstruction procedure based on the Feldkamp's volumetric cone-beam algorithm. The source used was of $40 \mathrm{kV}$ with an intensity of $22 \mu \mathrm{A}$, the rotation step $0.20^{\circ}$ and the frame averaging of 2 with a random movement of 10 .

The reserved sample cubes were cut into a smaller parallelepiped piece of $3.5 \times 1.8 \times 2.3 \mathrm{~mm}$ each to fit in the $\mu \mathrm{CT}$ device. We got 533 images of $1052 \times 1004$ pixels each with an intensity of 16 bits per pixel. The spatial resolution of the scanning is $4.4 \mu \mathrm{m}$ per pixel. The images show four structures: the exterior, two different density solid regions and the porous region (see Figure $2 \mathrm{~A}$ ). The images exhibit ring artefacts. We removed them by applying the method proposed by Sijbers and Postnov (2004) that consists of transforming the image into polar coordinates taking the ring artefacts as the noise centre, removing the now lineal artefacts and back transforming the filtered images.

With the set of piled images, we constructed a 3D virtual model composed of adjacent cubical cells, called voxels, such that each voxel vertex stores the grey level of the corresponding image pixel. We then performed a preprocessing stage before running the two mentioned numerical methods:

a) Conversion to 8 bits, after checking that the actual number of grey intensity levels in the image was about 256.

b) Computation of the segmentation threshold.

c) Binarization of the model according to the threshold.

The computation of the segmentation threshold is the most difficult step. In the binary segmentation, voxels are assigned either as belonging to the solid region or to the porous one depending on their value in comparison to the threshold. For voxels having a significantly high value, the classification is clear: they belong to the solid region. Analogously, low valued voxels belong to the porous region. However, there is some uncertainty in the classification of voxels having values near the threshold level and that contain a mixture of multiple tissue values (partial volume effect). These voxels are at the pores boundary or correspond to sub-voxel pores. This uncertainty yields to 
underestimation or overestimation of the pore boundaries and thus, to errors in the pores diameters estimation. Even more, the boundary between two adjacent pores can be broken forming one large pore, or, on the contrary, fictitious boundaries can be created. Figure 3 illustrates this phenomenon in two-dimensions. It represents two pores of different radius, both smaller than a pixel (Figure 3A), that, after scanning, correspond to two pixels of different grey level (Figure 3B). Depending on the segmentation threshold, both pixels are considered as belonging to the porous region (Figure $3 \mathrm{C}$ ), or only the darker one (Figure 3D), and even, none of them. In the former case (Figure 3C), since the two pixels are adjacent, the pore reconstruction step will not be able to detect if there are two adjacent pores or one large pore. With the estimated threshold, we obtained a binary model with a porosity similar to the global porosity measured by MIP.

The computation of the pores distribution with numerical methods is very memory and time consuming (from half an hour to three hours depending on the method). Therefore, we subdivided each voxel model into smaller submodels each of them at most of 200 voxels per axis, reducing the computation time to at most 9 minutes. We applied the numerical methods to each submodel separately and compared them. They didn't exhibit significant differences, since the samples were very homogeneous. In this paper, we show the data corresponding to a central submodel. Figure 4 shows a rendered view of three submodels, each corresponding to a different sample.

\subsection{Virtual porosimeter numerical method (VP)}

The input of this method is the voxel model and a list of decreasing diameters, $d_{1}, d_{2}, \ldots d_{n}$. that can be computed from the corresponding given pressures, used in the MIP. Each of these diameters, $d_{i}$, actually represents the diameter interval $\left[d_{i}, d_{i+1}\right]$. The output of the process is a labelled voxel model, each label indicating the associated diameter (Vergés et al., 2008 a).

The algorithm performs the following steps:

- Computation of a 2D digital skeleton with the same topology as the original model and with distance-labelled voxels (Ayala et al., 2007).

- for each $d_{i}$ :

- Selection of the skeleton voxels that are connected with the exterior or that are terminal voxels of a previous iteration, with diameter larger or equal to $d_{i}$ (seeds)

- Rasterization of a ball centred at each seed, labelling its voxels with the seed diameter. When overlapping, choose the larger diameter.

- Traversal of all skeleton voxels 26-neighbours of the current seed that have not been yet visited. For each of them, if its diameter is larger or equal to $d_{i}$, then process it in the same way as the seed. Else, label it as terminal voxel.

At the end of the process, any connected set of voxels with the same label is considered a pore. A graph is then devised in which nodes correspond to pores and edges are considered between any pair of adjacent pores. Two pores are adjacent is there exist a pair of voxels, each belonging to each pore, which are adjacent. 


\subsection{Pore-Topology Tracker numerical method (PTT)}

This approach is intended to represent the internal geometry of the porous space as a set of disjoint pores fulfilling specific shape restrictions (Vergés et al., $2008 \mathrm{~b}$ ). The method computes the set of maximal overlapping spheres that cover all the porous region. Pores are constructed as disjoint unions and differences of maximal spheres. The output is also a graph.

The algorithm performs the following steps:

- Computation of a 2D digital skeleton as the local maxima of the distance map. This is a non-topology preserving, reconstructible skeleton.

- For each sphere centred at a local maxima, in descending diameter order, not yet visited:

- Selection of all the spheres overlapping it

- Test considering the size and overlapping ratio in order to determine if the tested sphere is a pore, or it is absorbed by other maximal sphere.

- Construction of the graph. Nodes correspond to pores and edges connect any two adjacent pores.

\subsection{Pore viewer}

We have designed a specific pore visualization application (VPVIEWER) that allows us to interactively explore the virtual model of the sample using the graph model computed with any of the two methods (Grau et al., 2010).

Users can interactively select a pore and query for the visualization of all the pores connected to this one through paths of a given length. A colour ramp is used to indicate the distance of the pores from the selected one. Transparency effects and clipping of pores smaller than a given diameter are also provided. Figure 5 shows an example of these illustrations for the three samples. Moreover, we are able to explore a particular path between two connected pores. To better show the path, we apply a visual cut-away of the solid region that occludes the path (see Figure 6). Figure 7 shows several virtual intrusion steps for sample DS10.

\section{Results and discussion}

Table 1 shows the global porosimetry results obtained with MIP for the three studied samples. All the samples show similar characteristics of their porosity, with pores diameters ranging in size from 60-90 $\mu \mathrm{m}$ to $0.01 \mu \mathrm{m}$, and total porosity values ranging from $12 \%$ to $16 \%$. Finally, we indicate the diameters corresponding to the maximum differential volume intrusion.

Tables 2 and 3 show the results of the numerical simulations obtained by VP and PTT methods respectively. We show global parameters of porosity, effective porosity and pore interconnectivity. The effective porosity is computed as the percentage of reachable pores. The pore interconnectivity measures the ratio between the effective porosity and the global porosity. In addition, the tables contain data that derive from the pore size distribution: minimum and maximum pore diameter and, for the VP method, the diameter corresponding to the maximum differential intrusion volume, and, for the PTT method, the diameter corresponding to the maximum volume. Figures 8,9 and 10 show the pore-size distribution curves obtained by the three methods for sample DS10. The equivalent curves for samples DS11 and DS12 are similar. 
The global parameters of porosity, effective porosity and pore interconnectivity are the same with VP as with PTT, because these parameters depend on the binary model and not on the pore detection method itself. The comparison of these parameters with those obtained with MIP yields to the same conclusions for the three samples. The porosity of the samples is well approximated by the numerical simulations. Unlike MIP, the numerical methods provide information of pores non-reachable from outside, and therefore, they allow to compute the effective porosity and pores interconnectivity. For sample DS10, the pore interconnectivity is large, but samples DS11 and DS12 have a larger volume of non-reachable pores. When exploring graphically the models, we could observe that this was due to the presence of some closed pores inside the samples. The capacity of providing this information and to allow graphical exploration of the models is one of the main advantages of the numerical methods.

Regarding the pore size distribution, the resolution of the scanned images is the key factor of the numerical methods, and it is also its main limitation. Since the resolution of the images is of $4.4 \mu \mathrm{m}$ per pixel, in the numerical methods, the minimum detectable pore diameter is $8.7 \mu \mathrm{m}$. In the case of MIP, the minimum detectable pore diameter is around $0.01 \mu \mathrm{m}$. However, as shown in Figure 8 , the peak of the curve of the differential volume intrusion divided by the differential pressure according to the pore size, which is probably the most relevant data, falls in the range of the image resolution in the three cases.

When comparing the results of VP and MIP, we observe differences. This is due to the fact that if we try to match the global porosity of the sample in the segmentation, all the porosity corresponding to pores smaller than a voxel that the numerical method cannot detect, should be compensated by enlarging the pores boundaries. This produces a more or less homogeneous redistribution of the porosity, resulting in a diameter distribution curve with a shape similar to that of the low threshold, but shifted towards larger diameters. As a consequence, the maximum differential intrusion diameter can be higher than with MIP. It is the case of sample DS11. However, the global shape of the VP curves is similar to that of MIP. These results make us confident in the applicability of the method for other porous structures.

Comparing the VP and the PTT curves, we observe that the detected pore diameters are within the same range, but that the distribution is rather different. This result was expected, since the theoretical basis of the two methods is different. Since the PTT method tracks the internal topology of the porous region by sphere filling, the resulting curves have a larger number of points: more pores of different diameters are detected than with mercury intrusion. This result is coherent with the visual impressions of the graphical exploration of the models which shows a larger diversity in the pores diameters than with MIP and VP. The value of the PTT method is to provide this insight in the topological structure. The curve increases in the $y$-scale because the volume of pores is larger with a high segmentation threshold than with a low one, and the shape of the curve also changes. Again, this is due to the nature of the PTT. We obtain also smaller minimum diameters, because in PTT, the diameters are computed as the diameters of the minimum inscribed sphere. 


\section{Conclusions}

In this paper, we have analysed and compared the porosity of three sandstone samples applying physical measurements with MIP and numerical methods based on 3D micro-scanned images. The two limiting factors of the experiment are the resolution of the images in relation to the pores size and the segmentation process. The porosity of the samples measured by MIP was approximately one half of sub-voxel size (sub-pixel) and the other half above it. We have only been able to simulate numerically this second half. For pores of a larger diameter than the voxel size, with the numerical method VP, we have obtained a good approximation of the maximum diameter, the maximum intrusion diameters and the general shape of the MIP curve. Moreover, with the PTT method we have got a better appreciation of the actual topological distribution of the pores. Finally, the graphical exploration of the model using illustrative visualization methods have greatly helped us to understand the data and to check visually the global correctness of the results.

We believe that this methodology could be applied successfully to other types of samples as far as their pore size is detectable with an image scanning device. Our future research focuses on reproducing this experiment on other samples with different porosity size and topology.

\section{Acknowledgements}

This work has been partially supported by the following national projects: MAT2005-07244-C03-03, CGL2007-66431-C02-02/BTE and TIN2008-02903 and by the project 02-1/07 of the Institute for Bioengineering of Catalonia (IBEC).

\section{References}

Abouesa, A. and Morad, S., 2009. An integrated study of diagenesis and depositional facies in tidal sandstones: Hawaz Formation (Middle Ordovician), Murzuq Basin, Libya. Journal of Petroleum Geology, 32, 39 66.

Acharya, R.C., Van der Zee, S. and Leijnse, A., 2004. Porosity-permeability properties generated with a new 2-parameter 3D hydraulic pore-network model for consolidated and unconsolidated porous media. Advances in water resources, 27, $707-723$.

Ayala, D., Vergara, E. and Vergés, E., 2007. Improved skeleton computation of an encoded volume. Proceedings of Eurographics 2007: 33 - 36.

Cnudde, V., Cwirzen, A., Masschaele, B. and Jacobs, P.J.S., 2009. Porosity and microstructure characterization of building stones and concretes. Engineering Geology, 103, 76 - 83.

Delerue, J.F., Lomov, S.V., Parnas, R.S., Verpoest, I. and Wevers, M., 2003. Pore network modeling of permeability for textile reinforcements. Polymer Composites, 24, $344-357$.

Grau, S., Ayala, D., Tost, D., Miño, N, Muñoz, F. and González, A., 2005. In-silice evaluation of bone implants. Proceedings of CEIG'05, pp. $245-248$

Grau, S., Vergés, E., Tost, D. and Ayala, D., 2010. Exploration of porous structures with illustrative visualizations. Computers and Graphics, 5, doi:10.1016/j.cag.2010.05.001.

Liang, Z., Ioannidis, M.A. and Chatzis, I., 2000. Geometric and Topological 
Analysis of Three-Dimensional Porous Media: Pore Space Partitioning Based on Morphological Skeletonization. Journal of Colloid and Interface Science, $221,13-24$.

Lindquist, W.B., Venkatarangan, A., Dunsmuir, J. and Wong, T., 2000. Pore and throat size distributions measured from synchrotron X-ray tomographic images of Fontainebleau sandstones. Journal of Geophysical Research, $105,21509-21527$.

Monga, O., Ngom, F.N. and Delerue, J.F., 2007. Representing geometric structures in 3D tomography soil images: Application to pore-space modeling. Computers Geosciences, 33, 1140 - 1161.

Oren, P. and Bakke, S., 2003. Reconstruction of Berea sandstone and pore-scale modelling of wettability effects. Journal of Petroleum Science and Engineering, 39, 177 - 199.

Ramos, E., Marzo, M., de Gibert, J. M., Tawengi, K. S., Khoja, A. A. and Bolatti, N. D., 2006. Stratigraphy and sedimentology of the Middle Ordovician Hawaz Formation (Murzuq Basin, Libya). A.A.P.G. Bulletin, 90, 1309 - 1336.

Sijbers, J. and Postnov, A., 2004, Reduction of ring artifacts in high resolution micro-CT reconstructions, Physics in Medicine and Biology, 49, 14, 247253.

Silin, D.B., Jin, G. and Patzek, T.W., 2004. Robust determination of the pore space morphology in sedimentary rocks. Journal of Petroleum Technology. May: 69-70. SPE\#86296

Stroeven, P., Sluys, L.J., Guo, Z. and Stroeven M. Virtual Reality studies of Concrete, Forma, 21, 227-242, 2006

Taud, H., Martinez-Angeles, R., Parrot, J.F. and Hernandez-Escobedo, L., 2005. Porosity estimation method by X-ray computed tomography, Journal of Petroleum Science and Engineering. 47: 209 - 217.

Tuan, H.S. and D.W. Hutmacher, D.W., 2005. Application of micro CT and computation modeling in bone tissue engineering. Computer-Aided Design, 37, $1151-1161$

Vergés, E., Ayala, D., Grau, S. and Tost, D., 2008a. Virtual Porosimeter. Computer-Aided Design and Applications, 5, 557 - 564

Vergés, E., Ayala, D., Grau, S. and Tost, D., 2008b. 3D reconstruction and quantification of porous structures. Computer \& Graphics, 32, 438 - 444.

Vogel, H.J., Tölke, J., Schulz, V.P., Krafczyk, M. and Roth, K., 2005. Comparison of a Lattice-Boltzmann Model, a Full-Morphology Model, and a Pore Network Model for Determining Capillary Pressure-Saturation Relationships. Vadose Zone Journal, 4, 380 - 388. 


\begin{tabular}{|l|l|l|l|}
\hline Sample & DS10 & DS11 & DS12 \\
\hline Porosity \% & 16.06 & 15.20 & 11.97 \\
\hline Minimum pore diameter (um) & 0.01 & 0.01 & 0.01 \\
\hline Maximum pore diameter (um) & 90 & 60 & 95 \\
\hline Maximum differential intrusion diameter (um) & 14 & 9 & 8 \\
\hline
\end{tabular}

Table 1.

\begin{tabular}{|l|l|l|l|}
\hline Sample & DS10 & DS11 & DS12 \\
\hline Porosity \% & 13.67 & 13.74 & 13.67 \\
\hline Effective porosity \% & 7.89 & 2.9 & 1.53 \\
\hline Pore interconnectivity \% & 91.8 & 55.4 & 43.8 \\
\hline Minimum pore diameter (um) & 8.7 & 8.7 & 8.7 \\
\hline Maximum pore diameter (um) & 130 & 90 & 72 \\
\hline Diameter of maximum differential intrusion (um) & 14 & 28 & 8.7 \\
\hline
\end{tabular}

\section{Table 2.}

\begin{tabular}{|l|l|l|l|}
\hline Sample & DS10 & DS11 & DS12 \\
\hline Porosity \% & 13.67 & 13.74 & 13.67 \\
\hline Effective porosity \% & 7.89 & 2.9 & 1.53 \\
\hline Pore interconnectivity \% & 91.8 & 55.4 & 43.8 \\
\hline Minimum pore diameter (um) & 5.2 & 5 & 5 \\
\hline Maximum pore diameter (um) & 110 & 102 & 84 \\
\hline Diameter of maximum volume (um) & 28 & 10 & 11 \\
\hline
\end{tabular}

Table 3. 

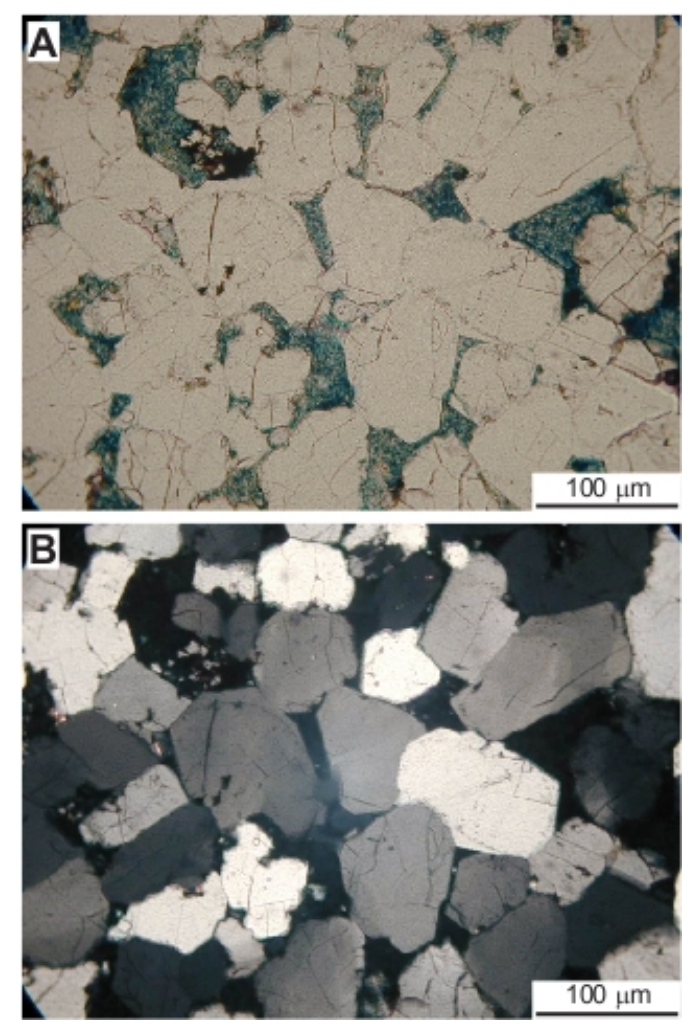

Figure 1.

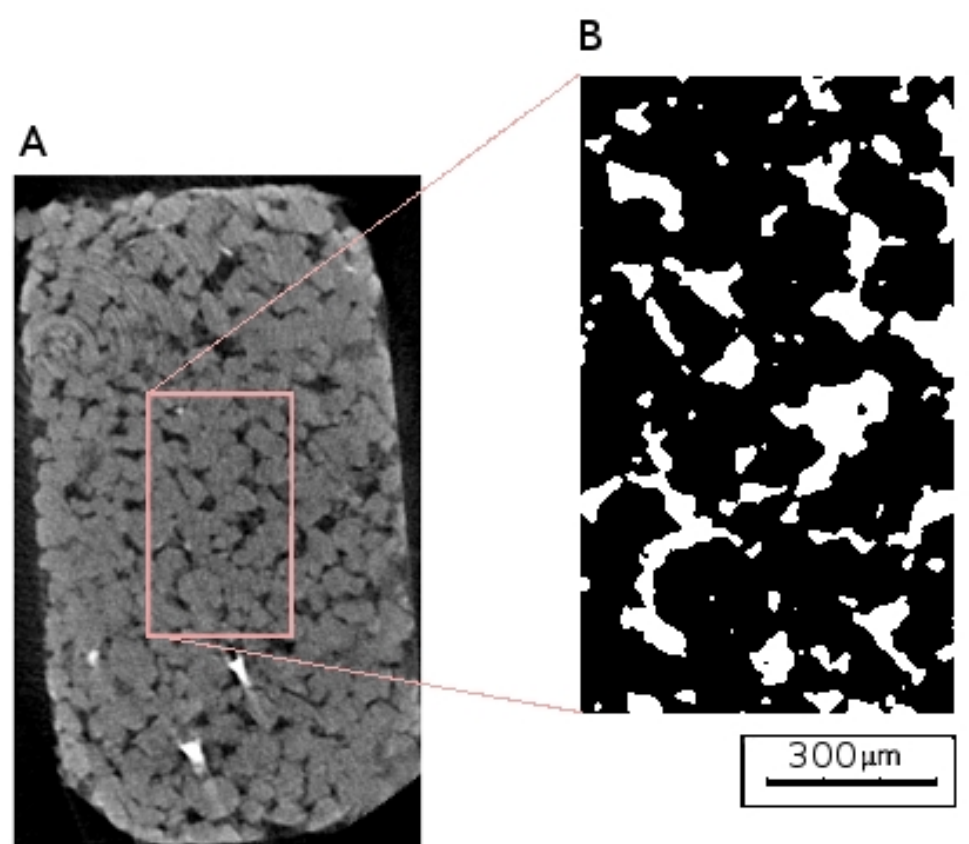

Figure 2. 

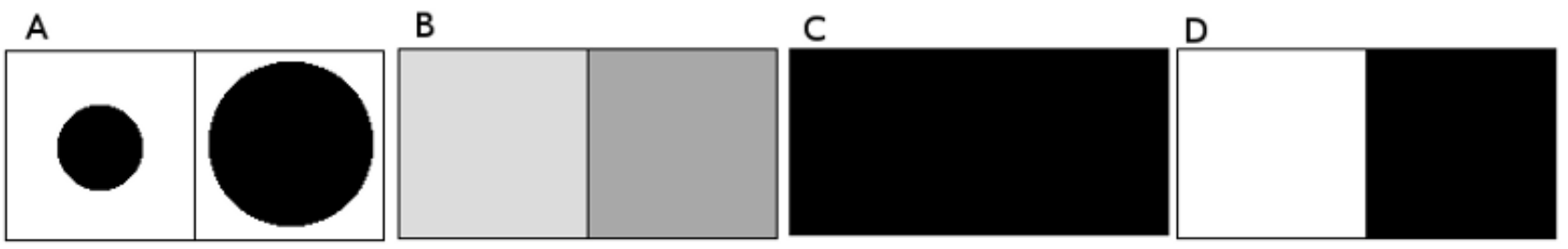

Figure 3.

A

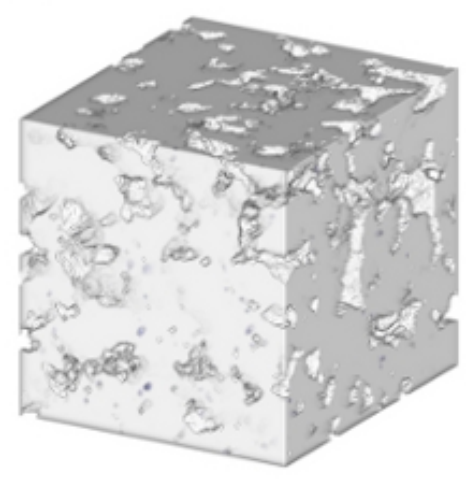

B

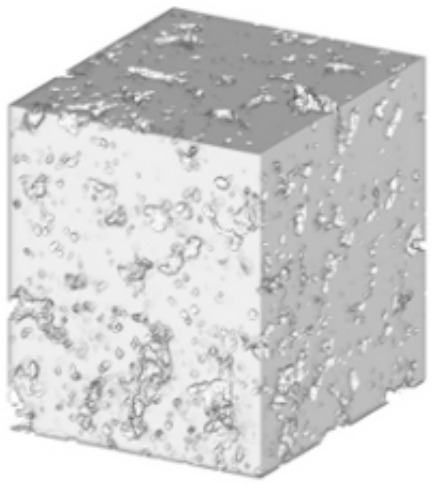

C

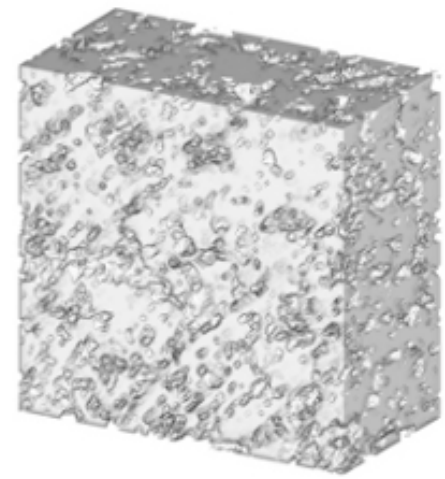

Figure 4.

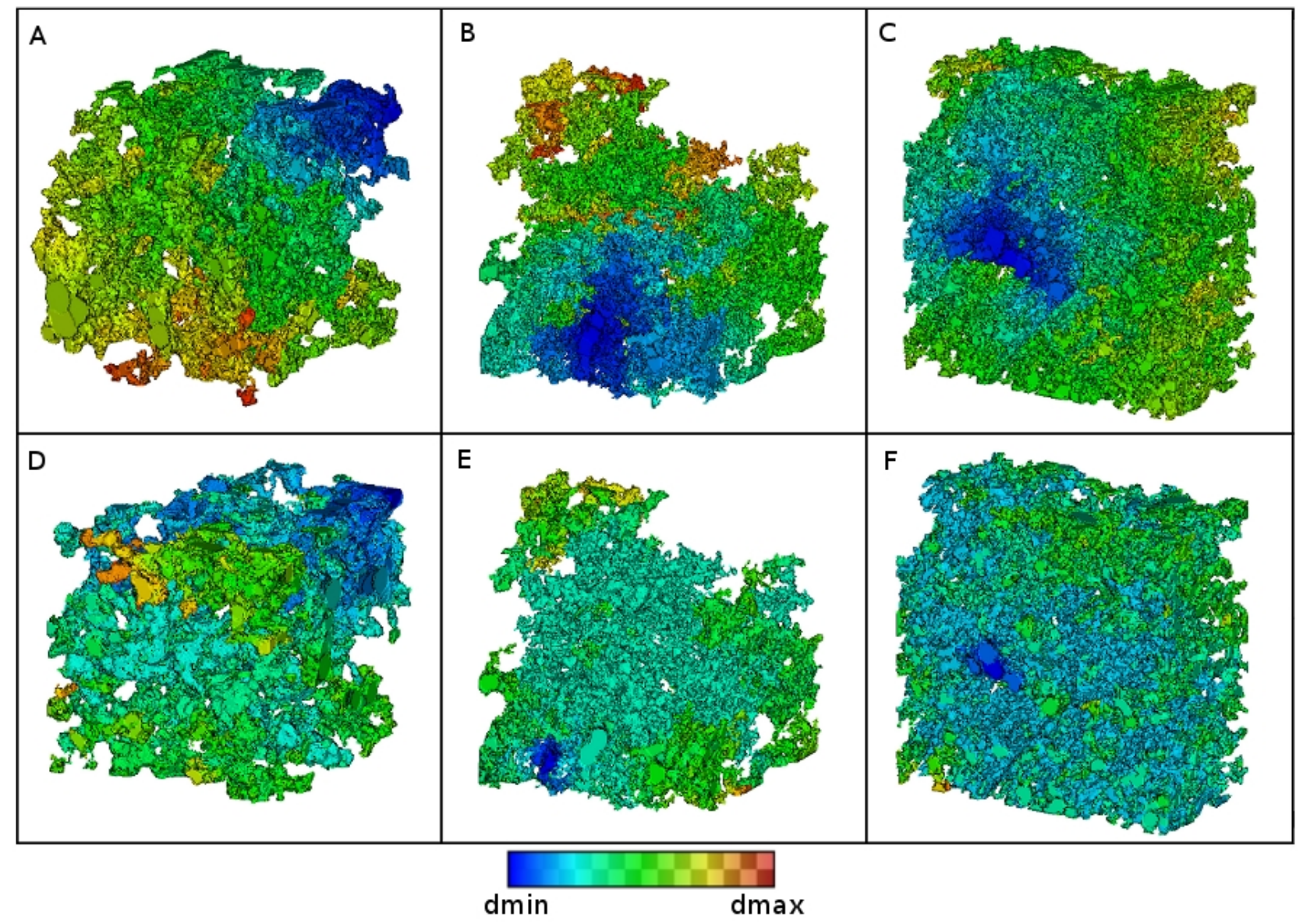

Figure 5. 


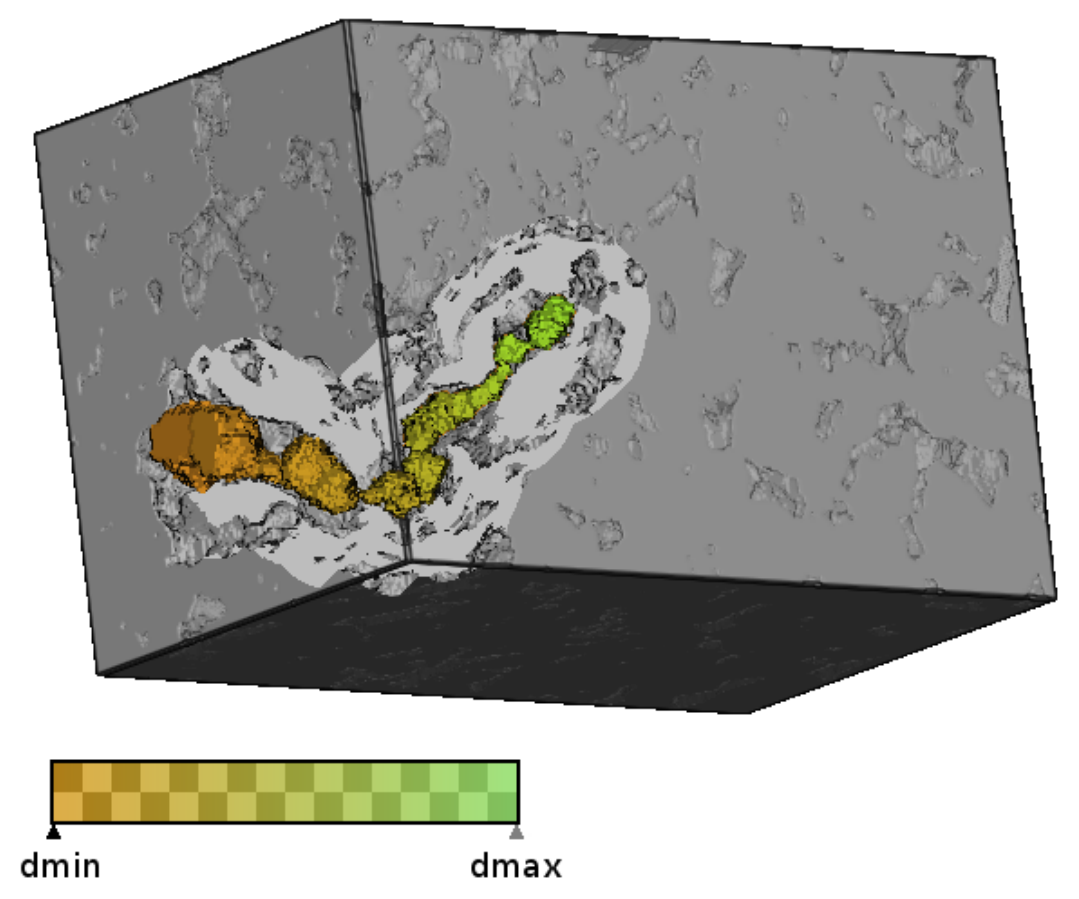

Figure 6.

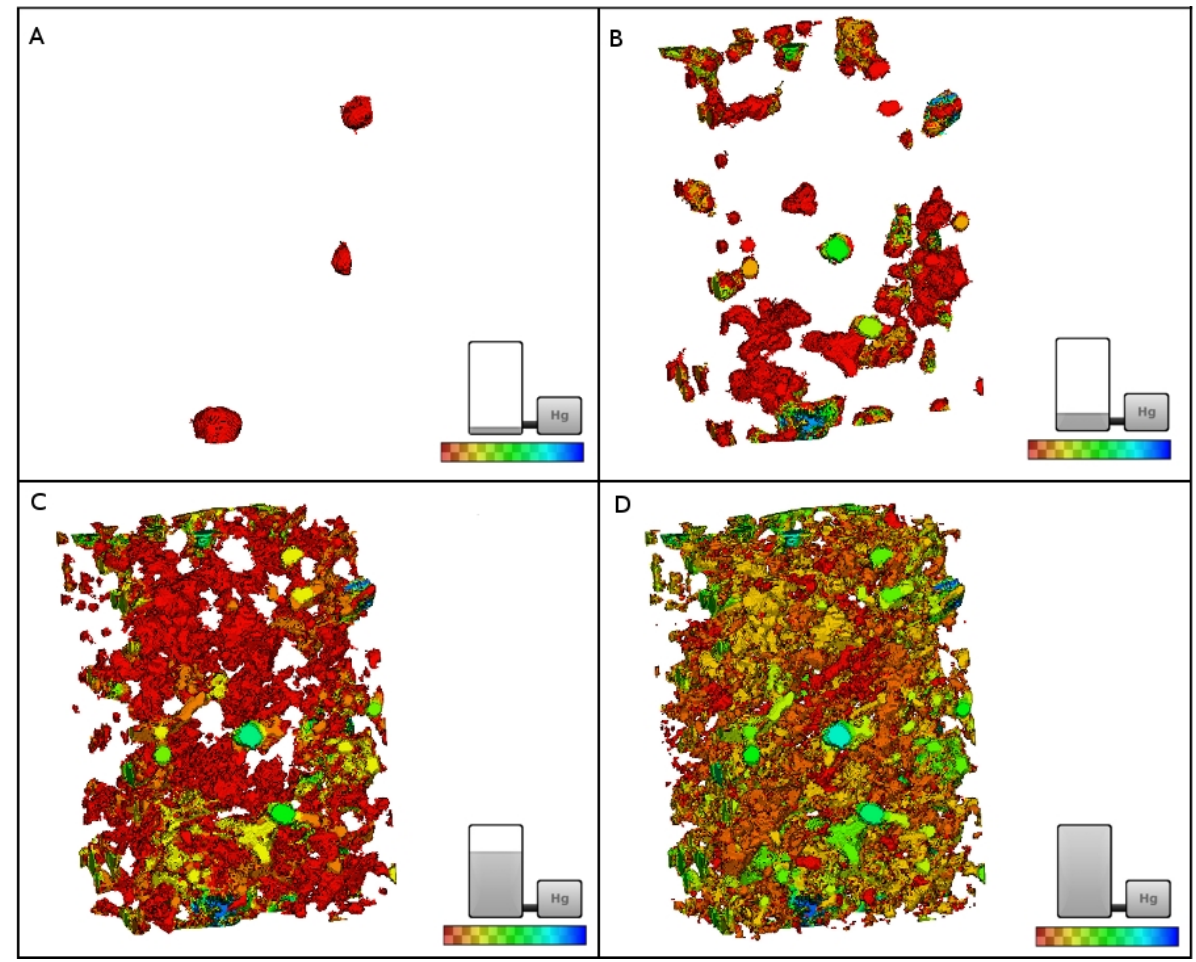

Figure 7. 
Pore Sizes for ds10 (MIP)

$\mathrm{dV} / \mathrm{dP}$ plot for ds 10

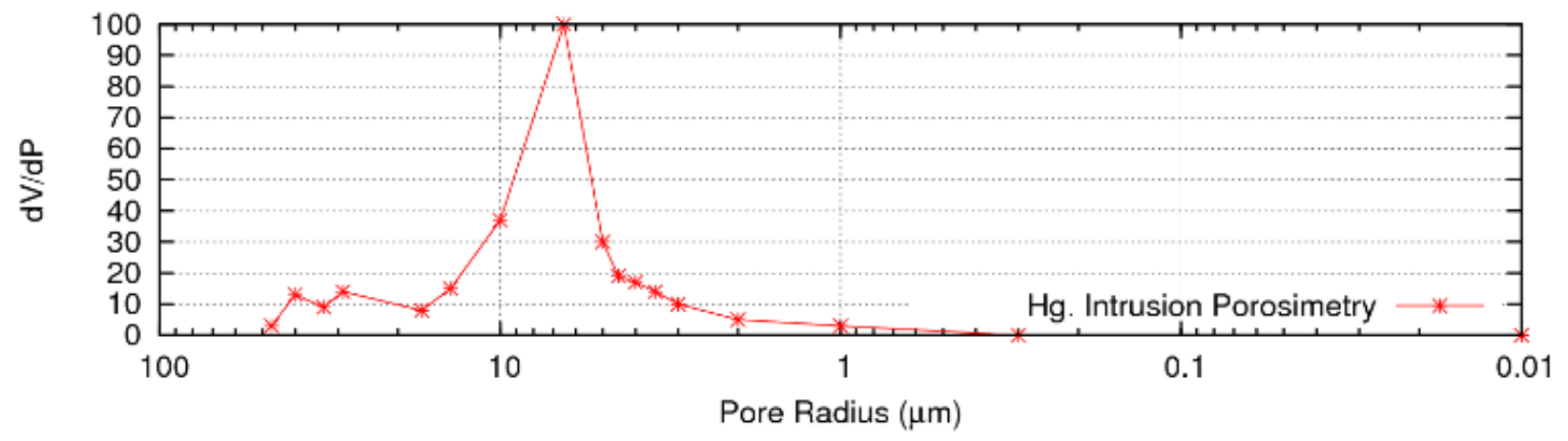

Relative accumulated volume plot for ds 10

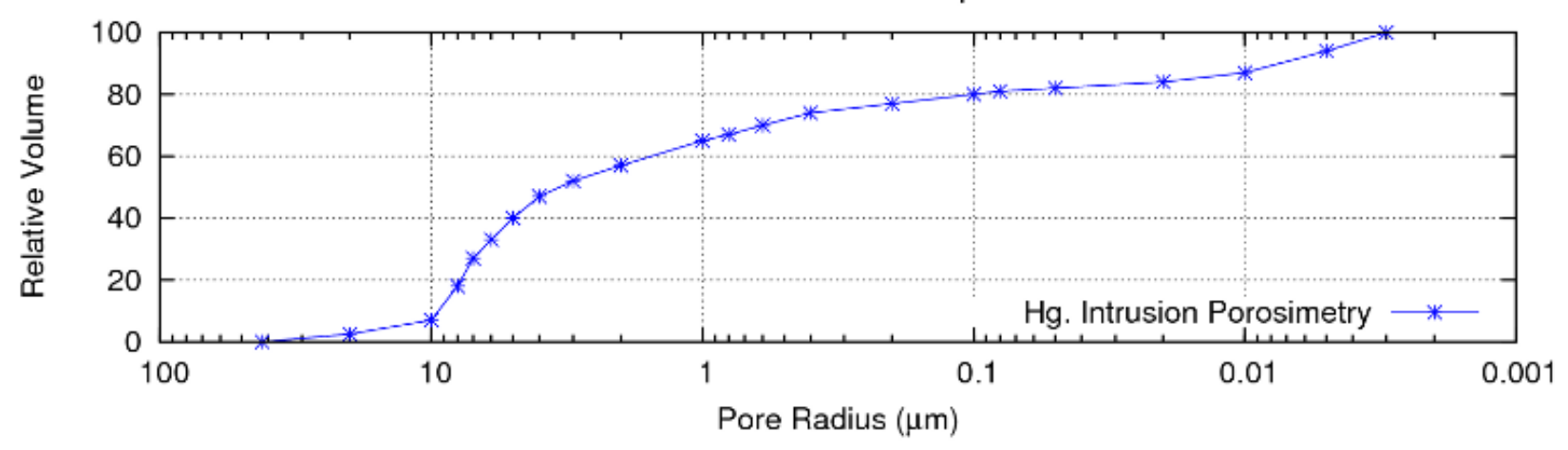

Figure 8.
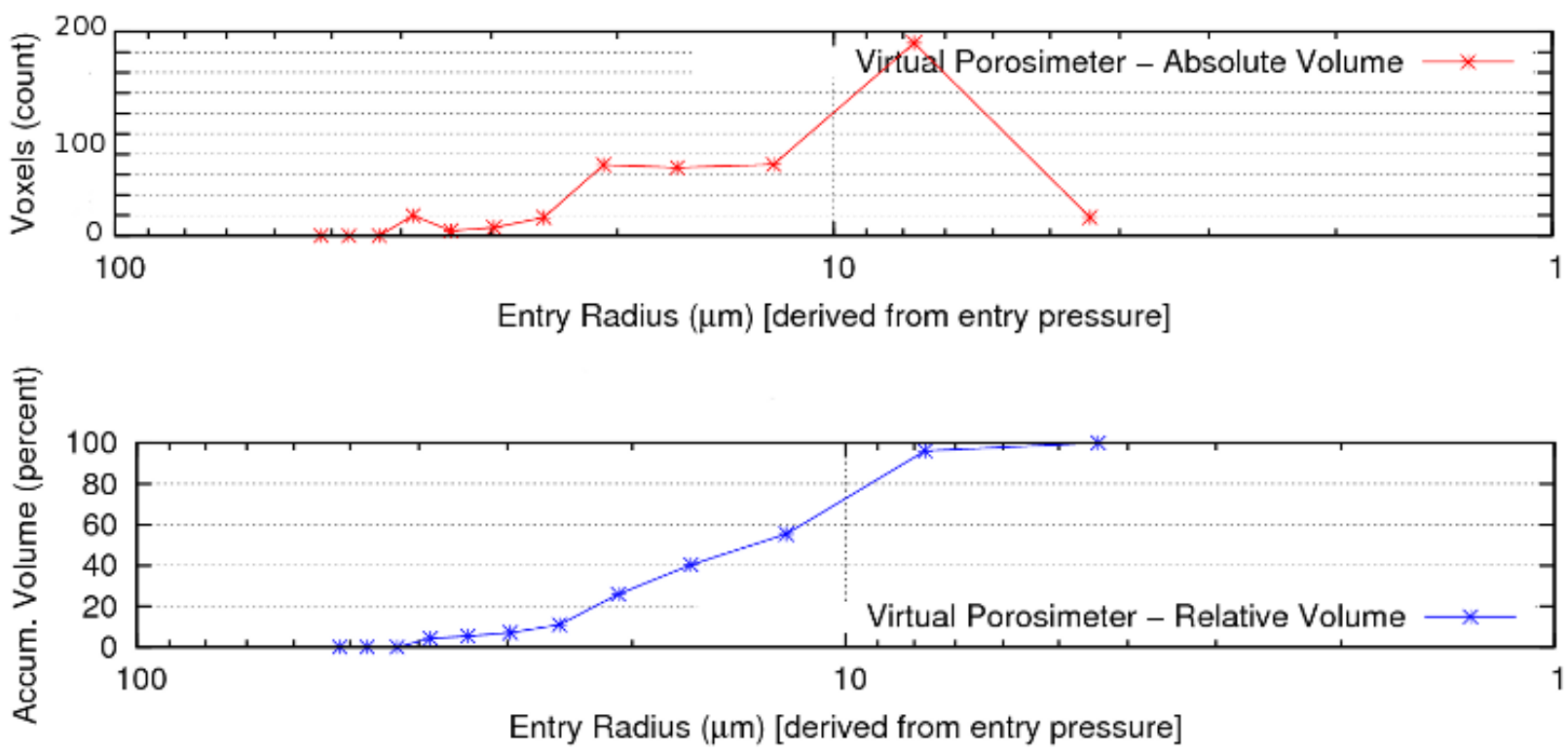

\section{Figure 9.}



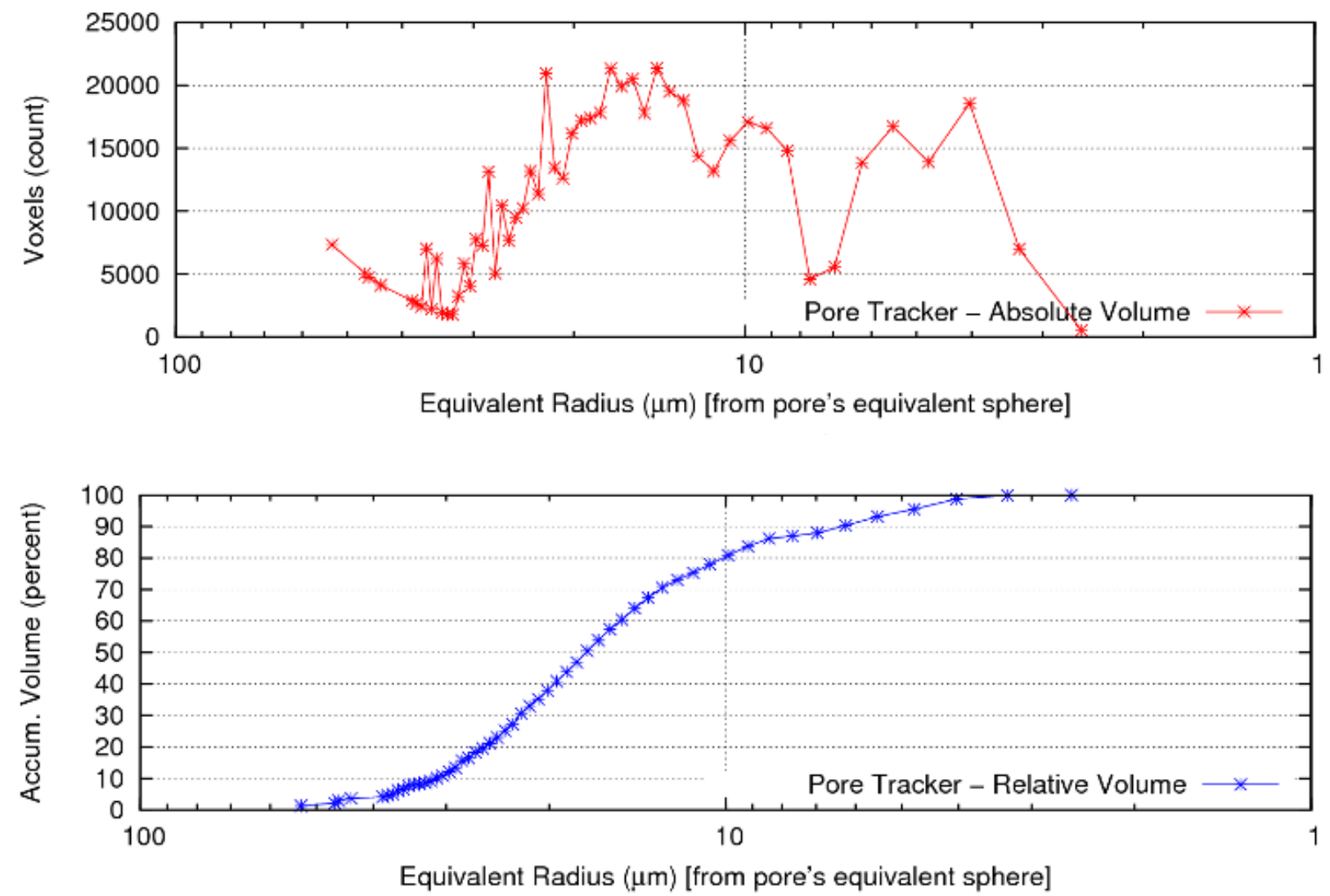

Figure 10. 